Maria Teresa Siniscalco*

ORCID: 0000-0001-5561-4614

Parigi, Francia

\title{
L'educando nello sguardo dell'educatore: spunti nel pensiero di Chiara Lubich e di Janusz Korczak
}

\author{
The Learner in the Eyes of the Educator: \\ Reflections on the Thought of Chiara Lubich \\ and Janusz Korczak
}

\begin{abstract}
Summary: The article aims to highlight some pedagogical implications of the text of Chiara Lubich Looking at All the Flowers, putting it in dialogue with the work of Janusz Korczak. The universality inherent in Chiara Lubich's exhortation to "look at all the flowers", can be found in the exhortation of Korczak to "respect all the ages of life", as a consequence of the high consideration that he has of the child and his rights, first of which the right to respect. The depth of meaning that "looking at" assumes in this text of Chiara Lubich, where it becomes synonymous with "loving", can be found declined and deployed in pedagogical terms in the work of Korczak.

* Maria Teresa Siniscalco, ha una laurea in Filosofia e un dottorato di ricerca in Pedagogia Sperimentale. Lavora per l'Istituto nazionale italiano per la valutazione del sistema educativo di istruzione e formazione come consulente nel campo della valutazione e della ricerca educativa. È stata il responsabile nazionale italiano di PISA 2003. Ha lavorato per l'International Association for the Evaluation of Educational Achievement (IEA) ad Amburgo. Collabora con EdU, Education for Unity. Contatto: Via Piave, 15, 00046 Grottaferrata (Rm). Italia. Mail: mtsiniscalco@aol.com
\end{abstract}


The juxtaposition of some passages of the texts of these two authors reveals profound assonances in the anthropological foundations of their thought.

Keywords: Chiara Lubich; Janusz Korczak; educator; learner; anthropology of education.

Sommario: L'articolo mira a evidenziare alcuni spunti pedagogici del testo di Chiara Lubich Guardare tutti i fiori, mettendoli in dialogo con il pensiero di Janusz Korczak. L'universalità insita nell'esortazione della Lubich a "guardare tutti i fiori", si ritrova nell'esortazione di Korczak a "rispettare tutte le età della vita", conseguenza della considerazione alta che egli ha del bambino e dei suoi diritti, primo dei quali il diritto al rispetto; lo spessore di significato che il "guardare" assume nel testo della Lubich, dove diventa sinonimo di "amare", lo si ritrova declinato e dispiegato in chiave educativa nell'opera di Korczak. L'accostamento di alcuni brani dei testi dei due autori rivela profonde assonanze nelle fondamenta antropologiche del loro pensiero.

Parole-chiavi: Chiara Lubich; Janusz Korczak; educatore; educando; antropologia dell'educazione.

Il presente articolo vuole mettere in relazione alcuni punti di un testo di Chiara Lubich - grande mistica cattolica del XX secolo e fondatrice del Movimento dei Focolari ${ }^{1}$ - Guardare tutti i fior ${ }^{2}$, scritto nel 1949, con l'esperienza e il pensiero dell'educatore, scrittore, medico e libero pensatore polacco

${ }^{1}$ Chiara Lubich nasce a Trento il 22 gennaio 1920 e muore a Rocca di Papa (Roma) il 14 marzo 2008. Nel 1943 si consacra a Dio e, in breve tempo, insieme alle prime persone che la seguono, dà vita a una corrente di rinnovamento spirituale e sociale animata dall'obiettivo di contribuire a costruire un mondo più unito e fraterno, in risposta alla preghiera di Gesù al Padre "Che tutti siano uno" (Gv 17, 21). Quello che prenderà il nome di Movimento dei Focolari si diffonde in pochi anni in tutta Italia, poi in Europa e nei cinque continenti, coinvolgendo persone di ogni età e di ogni orizzonte religioso e culturale [Chiara Lubich, La dottrina spirituale, ed. Michel Vandeleene (Milano: Mondadori, 2001)].

${ }^{2}$ Chiara Lubich, "Paradiso '49. Guardare tutti i fiori, 6 novembre 1949", in: Lucia Abignente, Maria Caterina Atzori et al., Guardare tutti i fiori, Studi della Scuola Abbà (Roma: Città Nuova Editrice, 2014), 10-12. 
Janusz Korczak ${ }^{3}$. Considereremo brevi frammenti dell'una e dell'altro, ben sapendo che ognuno di questi frammenti è come una tessera di rispettivi mosaici, ben più ampi e densi di significati. Queste brevi pagine, in altre parole, possono aprire un orizzonte tutto da indagare.

La lettura in chiave pedagogica del testo della Lubich - testo fondativo della "spiritualità collettiva" di cui l'autrice è stata l'origine ${ }^{4}$ dando vita al Movimento dei Focolari - per giustapporlo al pensiero di Korczak, è legittimata da diverse considerazioni. In primo luogo l'intero Movimento dei Focolari ha in sé una dimensione educativa, come è stato riconosciuto dalla sua stessa fondatrice: "Il nostro Movimento e la nostra storia, infatti, possono essere visti come un grande, straordinario evento educativo" luogo per la Lubich " "la spiritualità' include ogni espressione della vita umana e sociale", compreso quindi l'ambito dell'educazione e della riflessione pedagogica $^{6}$; in terzo luogo vi sono alcuni scritti dell'autrice che trattano esplicitamente di temi pedagogici; e infine vi è il fatto che la Lubich stessa è stata maestra nella scuola primaria, all'inizio della sua vita adulta, e ha continuato a esercitare le doti di cui aveva dato prova come giovane insegnante, nel corso della sua intera vita, come fondatrice e presidente di un movimento di estensione mondiale e di portata universale.

Perché Janusz Korczak in dialogo con questo testo della Lubich? Ebreo Polacco, nato e vissuto a Varsavia a cavallo tra il 19esimo e il 20esimo secolo, definito da Bruno Bettelheim "uno dei più grandi educatori di tutti i tempi" Korczak ha esercitato il suo essere educatore come una vocazione, occupan-

3 Nasce a Varsavia il 22 luglio del 1878 (o 1879) e muore a Treblinka nell'agosto del 1942. Janusz Korczak è lo pseudonimo con cui è più conosciuto, ma il suo vero nome è Henryk Goldszmit.

${ }^{4}$ Fabio Ciardi, "L'intuizione di una "spiritualità collettiva": un'unità di anime che rispecchia la Trinità di Lassù", in: Lucia Abignente, Maria Caterina Atzori et al., Guardare tutti i fiori. Da una pagina del '49 di Chiara Lubich. Studi della Scuola Abbà (Roma: Città Nuova Editrice, 2014), 25-38.

${ }_{5}$ Chiara Lubich, "Laurea honoris causa in Pedagogia", Washington D.C., 10 Novembre 2000, in: Chiara Lubich, Una cultura nuova per una società nuova (Roma: Città Nuova, 2002), 167. La dimensione educativa dell'opera di Chiara Lubich e del Movimento dei Focolari è testimoniata da diversi riconoscimenti pubblici, tra i quali - oltre alla Laurea honoris causa in Pedagogia conferita alla Lubich nel 2000 dalla Catholic University of America - vi è il conferimento del Premio Unesco per l'educazione alla Pace (Parigi, 17 dicembre 1996).

${ }^{6}$ Ciardi, "L'intuizione", 25.

${ }^{7}$ Bruno Bettelheim, "Prefazione", in: Janusz Korczak, Come amare il bambino (Milano: Luni, 2015), 7. 
dosi dei bambini ebrei di un orfanotrofio da lui creato nel 1912 e diretto per 30 anni - La casa degli orfani - e a partire dal 1919 collaborando anche alla gestione di un orfanotrofio per bambini cattolici, Casa Nostra. Come Maria Montessori e pochi altri all'inizio del XX secolo, Korczak - grande ammiratore di Pestalozzi - è stato l'autore di una rivoluzione nel modo di guardare al bambino: ci ha insegnato a guardare alla figura del bambino come persona dotata di piena dignità, avente "diritto al rispetto" pieno da parte degli adulti. Infine Korczak ha liberamente scelto di condividere la sorte degli orfani che aveva in custodia, quando questi sono stati deportati a Treblinka, dove è morto insieme a loro nel 1942.

Il lavoro e il pensiero di Korczak, così come la spiritualità di comunione della Lubich, nascono sullo sfondo di tempi duri, la prima guerra mondiale e l'affermarsi del nazismo per Korczak e la seconda guerra mondiale per Lubich. Il lavoro di educatore di Korczak, come l'intera opera della Lubich, testimoniano una straordinaria coerenza e compenetrazione tra pensiero e vita.

Nelle prossime pagine si partirà da due elementi del testo della Lubich: l'esortazione a "guardare tutti i fiori" e l'esplicitazione di che cosa significhi "guardare". Questi elementi verranno messi in relazione con aspetti del pensiero e dell'esperienza di Korczak, tenendo conto che i testi messi in dialogo hanno contesti e sviluppi propri, ma nella convinzione che possa essere arricchente vederne le reciproche assonanze.

\section{Perché "tutti i fiori"?}

Nel capoverso 933 del testo della Lubich leggiamo:

Dio chiede a noi di guardare tutti i fiori perché in tutti è Lui e solo osservandoli tutti si ama più Lui che i singoli fiori ${ }^{8}$.

Cominciamo dalla metafora del fiore e del giardino. Come ha evidenziato M. Caterina Atzori ${ }^{9}$, con questa metafora - che ha echi nella letteratura di tanti popoli - la Lubich si ispira probabilmente alle passeggiate in montagna

${ }^{8}$ Lubich, "Paradiso '49", 10 (v. 933).

9 Maria Caterina Atzori, "Guardare tutti i fiori: il contesto dello scritto chiariano", in: Lucia Abignente, Maria Caterina Atzori et al., Guardare tutti i fiori. Da una pagina del '49 di Chiara Lubich. Studi della Scuola Abbà (Roma: Città Nuova Editrice, 2014), 19-20. 
che segnano il periodo in cui scrive questo testo, durante l'estate del '49, e usa un'immagine - quella del giardino - vicina all'esperienza comune, per farsi capire da tutti, con la capacità comunicativa e didattica che la contraddistingue. Il "giardino fiorito" "è l'umanità vista con gli occhi di Dio, un'umanità dove la Sua presenza è resa viva dall'amore scambievole"10.

Perché questa esortazione a "guardare tutti i fiori"? Lubich spiega che il guardare tutti i fiori permette di vedere ogni singolo fiore nella sua essenza più profonda, che è la presenza di Dio in lui. Per avere lo stesso sguardo verso tutti è necessario mettere da parte se stessi, le proprie inclinazioni e simpatie, i propri interessi, per avere "una visione dall'Alto, dall'Uno, dal Vertice, da Dio"11, fino a scorgere nell'altro, in ciascuno, quella parte/presenza di luce che rivela la comune paternità di Dio. Lubich ci dice che il guardare, l'osservare tutti i fiori è la garanzia che in ciascuno si guardi/ami Dio, invece che il singolo fiore o sé stessi.

Qual è infatti l'alternativa al guardare tutti i fiori? Lubich la descrive come il guardare e l'ammirare un solo fiore: è quello che fanno coloro che "cercano Dio in loro"12. Guardare un solo fiore significa concentrarsi su Dio dentro di sé, amarLo solo in sé, e questo - dice Chiara - "non è ragionevole" perché è un amore che ha "ancora qualcosa di personale, d'egoistico"13.

Anche Korczak ricorre all'immagine del fiore, che per lui simboleggia il bambino, contrapponendolo al frutto, che simboleggia l'adulto, e anche lui chiama in causa Dio - oltre alla legge - a fondamento di uno sguardo corretto sul bambino che si traduce nel rispetto verso di lui: "Tutto ciò che è giovane ha piccolo valore commerciale: solo di fronte alla legge e a Dio, il fiore di melo e il grano in erba valgono quanto una mela o un campo di grano maturo"14. Il fiore qui è simbolo di una categoria, quella di chi è più piccolo, debole, dipendente, giovane, che - proprio per questo - agli occhi dell'adulto ha meno valore.

Korczak denuncia lo sguardo dell'adulto che confonde le dimensioni fisiche con la natura profonda e il valore: "Bisogna essere grandi, occupare un bel po' di posto, per suscitare stima e ammirazione. Piccolo vuol sempre

\footnotetext{
${ }^{10}$ Ibidem, 23.

${ }^{11}$ Lubich, "Paradiso '49", 10 (v. 930).

12 Ibidem, 10 (v. 931).

${ }^{13}$ Ibidem, 11 (v. 935).

${ }_{14}$ Janusz Korczak, Il diritto del bambino al rispetto (Milano: Luni, 1994), 33 (titolo originale Prawo dziecka do szacunku, 1929).
} 
dire banale, sprovvisto di interesse. [...] Un bambino, è così piccolo, così leggero... così poca cosa. Bisogna piegarsi, abbassarsi fino a lui”"

Il punto di vista di Korczak è diametralmente opposto a questo ed è ben sintetizzato nel prologo al lettore adulto del suo romanzo per bambini e adulti Quando ridiventerò bambino, dove capovolge la prospettiva corrente parlando dello sforzo richiesto all'adulto per avvicinarsi al bambino.

Dite:

È faticoso frequentare i bambini.

Avete ragione.

Poi aggiungete:

Perché bisogna mettersi al loro livello, abbassarsi, inclinarsi, curvarsi, farsi piccolo.

Ora avete torto.

Non è questo che più stanca. È piuttosto il fatto di essere obbligati a innalzarsi fino all'altezza dei loro sentimenti.

Tirarsi, allungarsi, alzarsi sulla punta dei piedi.

Per non ferirli ${ }^{16}$.

Al contrario di chi sottovaluta il bambino, precludendosi così la possibilità di capirlo e conoscerlo veramente, Korczak afferma che il bambino supera l'adulto nella sfera dei sentimenti, mentre sul piano intellettuale non se ne differenzia, quello che gli manca essendo piuttosto l'esperienza.

Anche Korczak, come la Lubich, menziona l'egoismo. Con grande acutezza osserva che l'adulto agisce in modo egoistico nei confronti del bambino non solo quando vuole farlo stare fermo e zitto per non essere disturbato, ma anche quando gli impone abbracci e coccole:

Siamo a tal punto sprovvisti di senso critico da scambiare per amicizia le carezze con cui opprimiamo i bambini? Non capiamo dunque che stringendo il bambino tra le nostre braccia cerchiamo in realtà di rifugiarci nelle sue, per sfuggire alle ore di sofferenza e di abbandono? [...] È egoismo non tenerezza.

Il primo diritto del bambino è il diritto al rispetto, cioè a essere riconosciuto come soggetto autonomo con una propria sensibilità e le proprie

15 Ibidem, 30-31.

16 Janusz Korczak, Quando ridiventerò bambino (Milano: Luni, 1995), 7 (titolo originale Kiedy znów będę maty, 1924). 
esigenze. Korczak declina il rispetto nei confronti del bambino, aiutandoci a capire la sua portata e le sue implicazioni. Si tratta di "rispetto per la sua ignoranza", "rispetto per la sua laboriosa ricerca di conoscenza", "rispetto per le sue sconfitte e le sue lacrime [...] che sono sempre il segno di una sofferenza", "rispetto per la proprietà e per il bilancio del bambino", "rispetto per i misteri e per i colpi che riserva il duro lavoro della crescita", "rispetto per i minuti del presente", in una parola "rispetto del diritto a essere com'è" ${ }^{17}$.

Il rispetto che l'adulto deve avere nei confronti del bambino, il rispetto che il bambino ha il diritto di ricevere dall'adulto, è al cuore del pensiero pedagogico di Korczak e della sua opera educativa, fondata sulla convinzione che i problemi educativi possano essere affrontati con successo solo con la partecipazione attiva dei bambini in quanto soggetti. Korczak è stato così un precursore dell'affermazione dei diritti del bambino ${ }^{18}$, anticipando di oltre 60 anni la Convenzione internazionale sui diritti dell'infanzia, approvata dall'Assemblea delle Nazioni Unite nel $1989^{19}$, per la quale il pensiero dell'educatore polacco costituirà un punto di riferimento.

Perché il bambino ha diritto a questo rispetto? Perché non c'è un'età che sia superiore all'altra. Scrive Korczak

A torto attribuiamo gradi diversi di maturità ai nostri poveri anni: non si può stabilire una gerarchia in base all'età [...] Quando parlo o gioco con un bambino, un istante della mia vita si unisce a un istante della sua e questi due istanti hanno la stessa maturità ${ }^{20}$.

Come evidenzia Bettelheim, "Korczak non ci insegna ad amare il bambino - cosa non difficile per quanto ancora troppo rara -, ma a rispettarlo e a comprenderlo a partire dai suoi punti di riferimento piuttosto che dai nostri" ${ }^{21}$, e così facendo egli supera quello che la Montessori ha definito l'“ego-

${ }^{17}$ Korczak, Il diritto, 56 e seguenti.

18 Tra le voci di chi, a cavallo tra il XIX e il XX secolo, ha cominciato a porre la questione dei diritti dell'infanzia, vi è Maria Montessori che, nel capitolo finale di Il segreto dell'infanzia intitolato appunto "I diritti del bambino", afferma: "di là di quel primo salutare risveglio non si trova in tutto il cammino della storia nessun fatto evidente che riveli qualche riconoscimento dei diritti del bambino o qualche intuizione della sua importanza" [Maria Montessori, Il segreto dell'infanzia (Milano: Garzanti, 1950), 299].

19 La Convenzione è preceduta dalla Dichiarazione sui diritti del bambino, promulgata dalle Nazioni Unite nel 1959.

${ }^{20}$ Korczak, Il diritto, 60.

${ }^{21}$ Bettelheim, "Prefazione", 7. 
centrismo" dell'adulto, che considera il bambino riferendosi a sé stesso, con il risultato di "una incomprensione sempre più profonda"22.

In sintonia con l'esortazione della Lubich a guardare/amare tutti i fiori in quel giardino che è l'umanità, l'educatore Korczak esorta a rispettare tutte le età della vita, come aventi la stessa maturità e lo stesso valore, al punto di affermare che la pedagogia ha fatto un errore a ritenersi come la scienza del bambino, mentre dovrebbe essere la scienza dell'uomo. Egli include così nel "tutti" che hanno diritto al più profondo rispetto anche gli esseri umani più piccoli, deboli, giovani e dipendenti $-\mathrm{i}$ bambini - e traduce questa sua convinzione in organizzazione e pratica educativa con gli ultimi degli ultimi, gli orfani degli orfanotrofi di cui ha la responsabilità, in un tempo in cui una tale sensibilità è ancora un'eccezione: grazie all'abolizione delle punizioni corporali (e praticamente delle punizioni tout court), alla presenza di un sistema di comunicazione bidirezionale tra il bambino e l'educatore attraverso la corrispondenza e alla creazione di un tribunale e di un parlamento - "nei quali" i giudici e i deputati sono i bambini stessi - il suo sistema educativo mira a dare vita a una società di bambini, organizzata secondo i principi di giustizia, di fratellanza, di eguaglianza nei diritti e nei doveri.

Sia nella Lubich, sia in Korczak l'attenzione e la tensione verso l'altro, verso tutti gli altri, compresi gli ultimi, è legata a una visione religiosa della persona umana e alla convinzione della presenza di Dio in lui. "Dio chiede a noi di guardare tutti i fiori [...] perché in tutti è Lui" $\mathrm{e}$ "Dio che è in me, che ha plasmato la mia anima, che vi riposa in Trinità con i santi e con gli Angeli, è anche nel cuore dei fratelli" ${ }^{\prime 23}$ scrive Lubich. Parlando del bambino appena nato, Korczak esprime l'idea della trascendenza insita nella persona umana con parole che mostrano il suo background scientifico e allo stesso tempo il suo animo di poeta e la sua sensibilità religiosa:

È talmente fragile da poter essere ucciso da un batterio visibile come un puntino solo dopo essere stato ingrandito mille volte [...]. Ma questo niente è fratello in tutto e per tutto dell'onda del mare, della raffica di vento, della saetta, del Sole, della Via Lattea. Questo pulviscolo è fratello della spiga di grano, dell'erba, della quercia, della palma, dei pulcini, dei piccoli di leone, del puledro, dei cuccioli di cane. Questo granello di sabbia abbraccia tutto con la mente: le stelle

${ }^{22}$ Montessori, Il segreto, 15.

${ }^{23}$ Lubich, "Paradiso '49", 11 (v. 933 e 934). 
e gli oceani, le montagne e gli abissi. E cosa è il contenuto dell'anima, se non l'universo illimitato? Ecco la contraddizione dell'esistenza umana, che sorge dalla polvere ed è abitata da $\mathrm{Dio}^{24}$.

Con parole diverse vi è l'affermazione della convinzione che "in tutti è Lui”, espressa dalla Lubich.

Korczak è animato dalla convinzione che l'uomo-bambino è un essere creato ed abitato da Dio e che il suo lavoro di educatore si fonda anche sull'“umile preghiera". Nella sua toccante Preghiera dell'educatore ci svela qualcosa del suo rapporto con Dio, come uomo e come educatore:

Non elevo a te lunghe preghiere, o Dio. Non ti invio numerosi sospiri...[...] e non ti chiedo doni per me [...] Ma ti chiedo: da' ai bambini una sorte buona, aiutali nello sforzo, benedici le loro fatiche. Conducili non per la via più facile, ma per quella più bella ${ }^{25}$.

\section{Che cosa significa "guardare"?}

Intrecciato con l'esortazione a guardare tutti i fiori nel testo di Chiara Lubich è il chiarimento di che cosa significhino i termini "guardare" e "osservare". Non si tratta semplicemente dell'atto di portare il proprio sguardo su qualcuno o qualcosa, di includerlo nel proprio campo di attenzione, ma è - allo stesso tempo in cui si fa questo e proprio perché lo sguardo non si limita al singolo, ma comprende "tutti" coloro che mi è dato di incontrare di amarlo, o meglio di amare Dio presente in lui:

e solo osservandoli tutti, si ama più Lui che i singoli fiori [...]. Non è ragionevole che io Lo ami solo in me...; [...] il mio Cielo è in me e come in me nell'anima dei fratelli. E come Lo amo in me, raccogliendomi in esso - quando sono sola - Lo amo nel fratello quando egli è presso di me"26.

24 Janusz Korczak, Come amare il bambino (Milano: Luni, 2015), 20.

${ }^{25}$ Il manoscritto di questa preghiera, scritta da Janusz Korczak il 27 aprile 1920, è stato conservato e ricopiato a mano da Maria Falkowska, e poi pubblicato in Maria Falkowska, Kalendarz życia, działalności i twórczości Janusza Korczaka (Warszawa: Nasza Księgarnia, 1989), 182, 208-209; e in: Janusz Korczak, Pisma wybrane, vol. 3 (Warszawa: Nasza Księgarnia, 1987), 403.

${ }^{26}$ Lubich, "Paradiso '49", 11 (v. 933, 935-936). 
Il "guardare" nel testo della Lubich risulta essere premessa e sinonimo al tempo stesso di "amare".

Qual è in Korczak il rapporto tra il guardare il bambino, il rispettare il suo diritto a essere così come è e l'amarlo? La sua opera pedagogica più importante si intitola Come amare il bambino. L'intero saggio, composto al suo interno da quattro parti, è il frutto dell'osservazione attenta e appassionata dei bambini e comincia proprio con l'invito a guardarli, a considerarli con attenzione: il guardare con attenzione il bambino, per Korczak, è la condizione e il primo passo per amarlo. In apertura alla prima parte, Il bambino in famiglia, chiarendo che non si tratta di un libro con "prescrizioni e ricette", Korczak si rivolge al lettore e chiarisce il suo pensiero su questo punto: "Mi preme che si capisca come nessun libro, nessun medico possono sostituire il proprio attento pensiero, la propria attenta percezione"27. Ritorna poi a più riprese su questa contrapposizione tra il cercare risposte nei libri e il guardare direttamente, che solo permette di capire proprio quel bambino, il proprio bambino: "I libri con le loro formule pronte, hanno offuscato la vista e impigrito la mente. Vivendo delle esperienze, delle analisi, delle opinioni altrui, i genitori hanno a tal punto perduto la fiducia in se stessi che non vogliono guardare con i propri occhi", e conclude con l'esortazione "Non dai libri, ma da noi stessi" 28 .

Il primo insegnamento di Korczak è che per imparare ad amare il bambino occorre fermarsi a osservarlo, lasciarsi interrogare da quanto si vede, e cercare da soli le risposte - a volte con fatica ed essendo coscienti che sono sempre parziali e provvisorie - ai propri interrogativi. L'invito a osservare il bambino viene ripetuto più volte nel corso del volume. Ad esempio dove egli mette in guardia dalle aspettative che i genitori hanno nei confronti dei figli: "Invece di osservare per conoscere e per sapere, si prende il primo esempio sottomano di «bambino ben riuscito» e si pretende dal proprio: «ecco il modello al quale devi somigliare»" ${ }^{29}$. Oppure quando parla della capacità di osservazione della madre di fronte ai primi segni di malattia del figlio, capacità a volte molto acuta, a volte inesistente: "Chiama pure il medico per una visita di dieci minuti, ma tu stessa tieni gli occhi aperti venti ore" 30 . Oppure ancora dove propone agli educatori, come "esercizio pratico", di osservare i bambini mentre fanno un girotondo ${ }^{31}$.

\footnotetext{
27 Korczak, Come amare, 18.

${ }^{28}$ Ibidem, 33 e 158.

29 Ibidem, 26.

${ }^{30}$ Ibidem, 33.

${ }^{31}$ Ibidem, 105.
} 
L'osservazione dei bambini è il primo strumento con cui Korczak si rapporta con loro e con il loro mondo. Per rendersene conto basta leggere ad esempio le annotazioni sul neonato o la descrizione di tanti gesti e reazioni apparentemente insignificanti dei bambini, che narrati da lui diventano pieni di senso, come i primi balbettamenti, nei quali riconosce "errori linguistici di grande logica che bisognerebbe trascrivere e rendere noti" 32 ; oppure le annotazioni sui diversi tipi di pianto del bambino, per non fare che due esempi. Lui stesso, nello scrivere del bambino, rivela qualcosa del proprio lavoro di osservazione. Ad esempio là dove dice che l'internato è il luogo dove è possibile "la microosservazione dei cambiamenti impercettibili" 33; o dove, fin dalla prima sera di una colonia estiva, racconta di aver preso appunti su ogni singolo bambino appena incontrato e registra di avere già qualcosa da annotare per la metà dei bambini. Anche la notte, quando tutti dormono nell'orfanotrofio, Korczak osserva i bambini nel sonno: "Sulla notte e sul sonno dei bambini ho degli appunti. Trentaquattro blocchetti di appunti" ${ }^{34}$. Il bambino è osservato nella maggior parte dei casi mentre interagisce con gli altri, il gruppo o gli educatori, perché è attraverso l'osservazione del suo essere in relazione che si può cogliere qualcosa della sua natura profonda. A proposito dei bambini impegnati in un gioco, ad esempio, Korczak scrive:

in ogni atto collettivo, quindi anche nel gioco, facendo la stessa cosa degli altri, ognuno si differenzia pur in un minuscolo particolare [...]. Da una conversazione confidenziale veniamo a sapere che cosa desidera, dalla sua osservazione nel gruppo sappiamo che cosa è in grado di realizzare; qui si comprende qual è il suo rapporto con la gente, là quali sono i motivi nascosti di tale rapporto. Se incontriamo un bambino unicamente quando è solo, lo conosciamo in modo unilaterale ${ }^{35}$.

Con queste premesse si può capire la finezza psicologica delle sue annotazioni sui bambini e sulla natura umana in generale. È impossibile darne qui anche solo un'idea. La ricchezza delle osservazioni si basa sulla consapevolezza che non ha senso parlare dei bambini come realtà uniforme, dal momento che è un mondo abitato da un'infinita diversità, da un bambino all'altro e anche - per lo stesso bambino - da un momento all'altro: "Per

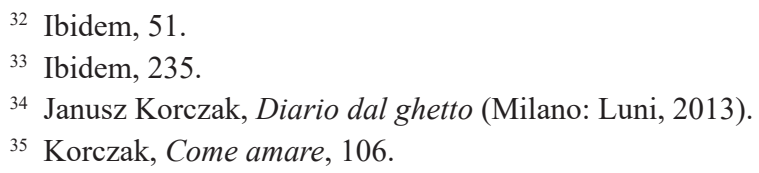

32 Ibidem, 51.

33 Ibidem, 235.

34 Janusz Korczak, Diario dal ghetto (Milano: Luni, 2013).

35 Korczak, Come amare, 106. 
me, e credo pure per ogni educatore, non esistono «i bambini», esistono gli individui, così diversi, così estremamente differenti, ciascuno dei quali reagisce in maniera così diversa e particolare a tutto ciò che lo circonda" ${ }^{36}$. "Sotto le divise tutte eguali battono cento cuori diversi e ognuno di essi rappresenta una diversa difficoltà, un diverso lavoro, differenti preoccupazioni e timori" ${ }^{37}$.

Accanto ai valori e alle virtù, Korczak dà un quadro lucido anche dei difetti dei bambini, parla delle nature più difficili e ostiche, che disturbano e in alcuni casi "rovinano" un gruppo e costituiscono sfide a volte difficilmente sormontabili per l'educatore. Di fronte a questi bambini, con il tempo Korczak impara a non aspettarsi cambiamenti irrealistici, a non chiedere promesse difficilmente mantenibili. Con grande saggezza, pone obiettivi ragionevoli, che - raggiunti - indicano il cammino per migliorare: "«Sei un impulsivo» dico a un ragazzo. «Va bene picchia pure, ma non troppo forte, arrabbiati, ma una sola volta al giorno». Se volete conoscere l'essenziale del mio metodo educativo, ecco, l'ho riassunto in questa frase" ${ }^{\prime 3}$.

Dalle sue pagine esce così una visione a tutto tondo del mondo infantile, disincantata e pure piena di misericordia. La misericordia si legge anche nelle strutture portanti del sistema educativo creato da Korczak nel suo orfanotrofio. Dei 110 paragrafi che compongono il codice del tribunale interno dell'orfanotrofio, 99 portano all'assoluzione: "Se qualcuno ha fatto qualcosa di male la cosa migliore è perdonarlo, aspettare finché non si sarà ravveduto". Gli ultimi 10 paragrafi invece prevedono una sanzione che va fino all'espulsione per tre mesi dall'istituto. Sulla stessa linea è uno dei giorni speciali del calendario istituito dal parlamento dei bambini: "il giorno dell'incoraggiamento". "Chi durante l'anno ha avuto dal tribunale il paragrafo di condanna più alto, riceve una sentenza assolutoria per le trasgressioni di tutta la settimana. [...] Il giorno dell'incoraggiamento viene introdotto per ricordare il caso di uno dei più cattivi soggetti che per una settimana non ha avuto neanche un processo" 39 . E altrove afferma che per uno sviluppo armonioso, aperto e libero dei bambini si dovrebbe fare loro una sola ingiunzione: "il comandamento: ama il tuo prossimo. Guarda il mondo intorno a te - sorridi" ${ }^{40}$.

\footnotetext{
36 Ibidem, 353.

${ }^{37}$ Ibidem, 173.

38 Ibidem, 73.

39 Ibidem, 356.

40 Ibidem, 170.
} 
Nell'osservazione, nella riflessione e nella pratica educativa Korczak è sostenuto certo dalla formazione scientifica di medico e dalla familiarità con l'osservazione clinica, ma anche e soprattutto da un infinito amore, stupore e rispetto per la natura umana nei bambini: "Oh, bacio questi bambini con lo sguardo, con il pensiero, con la domanda: che cosa siete, voi, mirabile mistero che cosa portate in voi?" ducatore secondo Korczak: mantenere lo sguardo attento, aperto e accogliente nei confronti della particolarità di ciascuno e alla novità di ogni giorno. Solo così l'educatore

amerà ogni bambino di un saggio amore, si interesserà alla sua vita spirituale, dei suoi bisogni, del suo destino. Più si avvicinerà al bambino più si accorgerà di caratteristiche degne di attenzione. Nella ricerca troverà sia la ricompensa sia lo stimolo per ulteriori ricerche, per ulteriori sforzi ${ }^{42}$.

Questi passaggi rispondono all'interrogativo iniziale. Anche nel caso di Korczak il guardare - l'osservare con attenzione e rispetto e il riflettere per capire l'altro - e l'amare sono profondamente interrelati. Anche se le parole sono diverse, sembrano crearsi le condizioni per vivere nella relazione educativa quanto la Lubich descrive con il linguaggio della mistica: "Occorre sì sempre raccogliersi anche in presenza del fratello, ma non sfuggendo la creatura, bensì raccogliendola nel proprio Cielo e raccogliendo sé nel suo Cielo"43.

\section{Alcuni cenni in guisa di conclusione}

In queste pagine si è appena sfiorato il denso testo della Lubich Guardare tutti i fiori e si è ripercorso solo sinteticamente e parzialmente il pensiero di Korczak, alla luce di due punti tratti da quel testo: il significato di "guardare", sinonimo di amare, e il richiamo all'universalità dell'amore, insito nell'esortazione a "guardare tutti i fiori".

Guardare. Nel testo della Lubich "guardare" e "amare" si alternano, risultando di fatto sinonimi. Analogamente, nella principale opera pedagogica

\footnotetext{
${ }^{41}$ Ibidem, 52.

42 Ibidem, 238.

${ }^{43}$ Lubich, "Paradiso '49", 11 (v. 938).
} 
di Korczak, Come amare il bambino, il guardare e l'amare il bambino sono saldati in modo indissolubile: occorre fermarsi a osservare con attenzione il bambino, il proprio bambino, per imparare ad amarlo; l'amore non è frutto di improvvisazione, né si può amare applicando ricette generali che vengono dall'esterno, ma è necessario fermarsi ad osservare il bambino, ciascun bambino, con attenzione, impegno, umiltà. Korczak educatore e pedagogista mostra come il guardare e l'imparare ad amare siano intimamente connessi.

Guardare tutti. La Lubich parla in nome di una visione del mondo dall'Alto, dall'Uno, che porta a guardare e amare tutti e in ciascuno Dio. Korczak invita analogamente - in campo educativo - a un capovolgimento di prospettiva, giungendo a dire che la pedagogia non dovrebbe essere la scienza del bambino, ma la scienza dell'uomo, perché tutte le età della vita hanno lo stesso valore, e ciascuna - inclusa l'infanzia - va compresa e rispettata a partire dai suoi propri punti di riferimento.

Mettere in dialogo il testo della Lubich con il pensiero di Korczak può sembrare forse non giustificato e arrischiato, essendo sempre in agguato il pericolo di cadere in forzature e semplificazioni. Tuttavia, alla luce del percorso che si è tracciato in queste pagine, ci sembra di poter affermare che il confronto è fecondo e, come si è visto, mette in luce profonde assonanze, che hanno le loro radici in una visione antropologica aperta alla trascendenza.

Per entrambi la natura più vera e profonda dell'essere umano, di ogni essere umano, può essere colta e incontrata davvero solo attraverso uno sguardo sensibile alla trascendenza. Con un linguaggio mistico e teologico la Lubich dice che l'incontro con l'altro è incontro "tra due Cieli [...] quello del Dio in me con quello di Dio nel fratello". Dal canto suo Korczak, con un linguaggio di volta in volta vicino a quello della scienza e a quello della poesia, parlando di un bambino appena nato, sottolinea la fragilità dell'essere umano, che può essere ucciso da un batterio invisibile, e allo stesso tempo la sua grandezza: ha un'anima che abbraccia l'universo ed è abitato da Dio.

L'accostamento di questi due autori ha permesso di evidenziare che, in un certo senso, in Korczak sono presenti tratti della verità luminosa espressa da Chiara sull'umanità, come giardino in cui ogni fiore nasconde e svela la presenza di Dio. La pagina di Chiara aiuta così a trovare nelle parole di Korczak una dimensione più profonda della sua visione educativa. Allo stesso tempo i testi di Korczak confermano la fecondità di una lettura di Chiara in chiave pedagogica, mettendone in luce la sintonia con il lavoro e il pensiero di un educatore di grande sensibilità, coerenza e attualità. 


\section{Bibliografia}

Atzori, Maria Caterina. "Guardare tutti i fiori: il contesto dello scritto chiariano". In: Lucia Abignente, Maria Caterina Atzori et al., Guardare tutti i fiori. Da una pagina del '49 di Chiara Lubich. Studi della Scuola Abbà, 19-20. Roma: Città Nuova Editrice, 2014.

Bettelheim, Bruno. "Prefazione". In: Janusz Korczak, Come amare il bambino, 7-8. Milano: Luni, 2015.

Ciardi, Fabio. "L'intuizione di una "spiritualità collettiva": un'unità di anime che rispecchia la Trinità di Lassù". In: Lucia Abignente, Maria Caterina Atzori et al., Guardare tutti i fiori. Da una pagina del '49 di Chiara Lubich. Studi della Scuola Abbà, 25-38. Roma: Città Nuova Editrice, 2014.

Falkowska, Maria. Kalendarz życia, dziatalności i twórczości Janusza Korczaka. Warszawa: Nasza Księgarnia, 1989.

Korczak, Janusz. Come amare il bambino. Milano: Luni, 2015.

Korczak, Janusz. Diario dal ghetto. Milano: Luni, 2013.

Korczak, Janusz. Pisma wybrane. Vol. 3. Warszawa: Nasza Księgarnia, 1987.

Korczak, Janusz. Il diritto del bambino al rispetto. Milano: Luni, 1994.

Korczak, Janusz. Quando ridiventerò bambino. Milano: Luni, 1995.

Lubich, Chiara. La dottrina spirituale, ed. Michel Vandeleene. Milano: Mondadori, 2001.

Lubich, Chiara. "Laurea honoris causa in Pedagogia". In: Chiara Lubich, Una cultura nuova per una società nuova, 161-173. Roma: Città Nuova, 2002.

Lubich, Chiara. "Paradiso '49. Guardare tutti i fiori, 6 novembre 1949". In: Lucia Abignente, Maria Caterina Atzori et al., Guardare tutti i fiori, Studi della Scuola Abbà, 10-12. Roma: Città Nuova Editrice, 2014.

Montessori, Maria. Il segreto dell'infanzia. Milano: Garzanti, 1950. 
